\title{
Heat and Photon Energy Phenomena: Dealing with Matter at Atomic and Electronic Level
}

\begin{abstract}
Mubarak Ali
Department of Physics, COMSATS University Islamabad, Islamabad Campus, Park Road, 45550, Pakistan, E-mail: mubarak74@mail.com, mubarak74@comsats.edu.pk
\end{abstract}

\begin{abstract}
Technology is reaching its climax, but the basic understanding of science in numerous phenomena is still required. A misconception in the use of terms, photon and electron, exists in different areas of the science. When an electron of the outer ring of silicon atom executes interstate dynamics for only one cycle, it generates the force and energy equal to the unit photon. So, interstate dynamics of the electron for one forward cycle and one reverse cycle generate the overt photon having the least measured length. Thus, a unit photon is a subset of the overt photon. When the photon of suitable length interacts with the side of laterally orientated electron clamped by the energy knot of atom, photon divides into the tits and bits of heat. When the photon interacts with the tip of laterally orientated electron by making the approximate angle of $90^{\circ}$, photon divides into the bits of energy having shape like integral symbols. In silicon atom, electrons of the outer ring execute confined interstate dynamics upon exerting forces along the poles; centre of the atom acts as the reference point for electrons executing interstate dynamics and the lateral-wise lengths of the electrons are along the northsouth poles. In neutral-state silicon atom, the involved heat energy starts wrapping the force shaping along the trajectory of electron executing interstate dynamics of forward and reverse cycles. The force is being shaped on the sides of electron not dealing with the exertion of forces. In interstate dynamics, electron of the outer ring first reaches to the 'maximum limit point', where energy of one bit engaged to wrap force shaping along the trajectory. From the 'maximum limit point', electron completes the second half cycle by dealing with the exerting forces, where again energy of one bit is engaged. In this way, an electron depicts the force and energy relation in the form of unit photon. The shape of unit photon is like Gaussian distribution turned from both ends. If the
\end{abstract}


uninterrupted supply of heat energy is available for neutral-state silicon atom, interstate dynamics of the electron generate the photon (energy) having the shape like wave. Path independent forces (but interstate dependent forces) take over the control of electron,

thus electron executes dynamics nearly in the speed of light. A photon can be in the continuous and unending length if the changing aspect of the electron remains uninterrupted. In the interstate dynamics, conservative forces exert to position-acquiring electron as per the natural viability. Electron of interstate dynamics recalls auxiliary moment of inertia at each point of turning as it does not have contact to energy knots confining the dynamics. Under neutral states, different atoms generate photon energy of different shapes. So, atoms change correspondence according to the mechanism of electron dynamics. Here, heat and photon energy discuss the matter at atomic and electronic levels to explore the foundation of science.

Keywords: Heat energy; Photons; Fundamental forces; Electron dynamics; Atomic scale phenomenon; Electronic scale phenomenon

\section{Introduction}

Creation has been benefited by heat and photon energy since its existence. Again, different phenomena such as electrical discharges, etc. have been the subject of many studies since long. Catching fire in different types of matter and burning of various commodities are the usual phenomena that have been under observation since the existence of life.

Many studies are available in the literature dealing with light-matter interaction, where it is mainly covered under a phenomenon known as surface plasmons. The origin of plasmons has been explored in some early published reports [1-4]. A plasmon is a quantum of plasma oscillating and representing the collective oscillations of the free electron gas density - a general definition extracted from the literature.

The interaction of light (photon) with matter is recognized in the form of various terminologies, such as phonon, excitons and plasmons. A reviewed study discussed light-matter interaction considering the properties of polariton modes in materials of twodimensional and their applications in a suitable range of spectrum [5]. The concept of 
excitons (electron-hole pairs) was first proposed by Frenkel [6]. It deals with an excited state of the atom (in a lattice) travelling in particle-like fashion without the net transfer of charge. Excitons can be formed due to the absorption of photon by a semiconductor (quantum dot) [7]; a phonon is a collective excitation in a periodic and elastic arrangement of atoms or molecules in the condensed matter.

A vast number of studies deal the different developing processes involving tiny-sized particles. The tiny-sized cluster is a simple chemical compound, which has a variety of important applications in diversified areas [8]. The unique nature of nanocrystals solicits the fabrication of new materials having the controlled features [9]. The likely development of nanoparticle technology is an obvious long-term benefit [10]. With the success of assembling colloidal matter in a useful structure, the atoms and molecules will be treated as materials in near future [11]. The investigation of the dynamics of individual nanoparticle should be taken as a prime concern prior to go for sound deliberations [12]. A good understanding about the surface features of nanoparticles will lead to the development of high order materials [13]. Tiny-sized clusters possess molecule-shaped electronic and non-face centered cubic geometric structures [14]. Chemical properties of gold nanoparticles change with size [15]. The development process of various tiny-sized and large-sized particles under varying concentration of gold precursor was studied while employing the pulse-based electron-photon/solution interface process [16]. It has been discussed that localized dynamics of the process is one of the causes that contributes to develop the structure of tiny-sized particles for gold [16-20], silver [20] and carbon [21, 22]. The study of tiny-shaped particle dealing with the elongation of atoms of arrays was also studied [23]. When the stretching of energy knots clamped electrons in an atom is unidirectionally based, it is related to the elongated process of that atom [24].

Sir Isaac Newton explained gravity, which is called Newtonian Physics. There is no such concept regarding the opposite of gravity in the Theory of General Relativity as explained by Sir Albert Einstein. Bohr proposed that electrons move around the nucleus in allocated orbits, where they have fixed energy if the atom is in ground state. Thus, a levitational force (exerting at the electron level) in atoms of suitable elements remains 
beyond understanding, and the study of band gap, valence band and conduction band prevails in all the related phenomena. The configuration of atomic structure remained only associated with the studies of orbits and shells. The concepts of atomic structures with quantum states are also studied.

A recently published study discussed the atomic structure in different gaseous and solid elements with new insight [25]. However, the conventional studies kept the researchers away from thinking about the force and energy behaviors of atoms. Huge efforts were made to explore the technological advances and breakthroughs. The efforts put forth toward exploring the basic and fundamental sciences remained less. Nevertheless, fundamental aspects of structure evolutions in suitable atoms were studied in the separate study [26]. Again, fundamental aspects of binding different state carbon atoms have been explored [27].

Here, interactions of photons with clamped energy knot electrons of the hypothesized solid atoms are discussed. Conversion of heat energy into the photon energy is clarified by considering the silicon atom as the model system. The exerting force to electrons of the outer ring of silicon atom is pinpointed. Photons of different shapes are also discussed. Analogy in the use of photon and electron is singled out.

\section{Results and Discussion}

When photons of characteristics current propagate in excessive population, flowing inert gas atoms split into the electron streams [24]; splitting electrons carry forcing energy of chasing photons and impingement of such electrons either elongate or deform the underneath solid atoms. So, solid atoms do not retain the behavior of original state. Solid atoms can also deform under the process of synergy, where interaction of the medium plays a vital role [16]; atoms deal with different sorts of interactions in the medium. Deformation of atoms of tiny-sized particles have been observed in colloids of gold, silver and the binary composition [16-20]. Deformation behavior of atoms has also been discussed in tiny grain carbon films [21, 22]. A uniform elongation behavior of gold atoms of tiny-shaped particle was discussed in a separate study [23]. 
Photonic current, also known as electric or electronic current, is stated when photons propagate through a wire ideally in the width of interstate electron gap of silicon atom in the outer ring [24]; photonic current does not infer the flow of electrons or charged particles. However, electrons of the suitable atoms can transform heat energy into the photon energy when executing the confined interstate dynamics. So, a photonic current is related to force, energy and the medium of propagation [24]; a photon keeps the features when propagates in the interstate electron gaps of suitable atoms.

For the solid atoms, the force and energy relationship shows a direct behavior, which is not the case in gaseous atoms [25]. Three different ground points of atoms belonging to suitable elements discussed where conservative forces involved to execute the confined interstate electron dynamics [26]. Depending on the state of carbon atom, different forces engage to form the different carbon structures [27].

These studies enlighten that energy and force together shape the photon. In atoms of the suitable element, an interstate gap of electron dynamics can determine the conserved nature of energy. Supplied heat energy to atoms can be utilized to generate photons under the confined interstate electron dynamics. When such photons propagates in the interstate electron gap of atoms, they can be related to the photonic current. So, the generated force and energy can be transported from generation point to consumption end. Instead of propagation, photons can travel when medium is the air.

\subsection{Heat energy - atomic and electronic scale phenomena}

When traveling photons interact with water have less forcing energy, they raise the temperature of the water to a less degree of warmness. When traveling photons interact with water have more forcing energy, they raise the temperature of the water to a greater degree of warmness. A long length (overt) photon carries more energy as compared to energy of the short length (overt) photon. Upon heating of the medium by entered photons, shaped force of the photons also gradually permeated into the medium. Once photons entered the water medium, they divided into the pieces. So, photons are no more with their nodes and antinodes. The heat energy of the pieces of photons dissipates in the water, so raise the temperature. The term 'partially 
propagation' can be employed here as the broken pieces of the unit photon are not fully static and the inertial property of the electron still exists in the auxiliary manner. In this context, broken pieces of different photons dissipate the heat when propagate and travel through the electronic gaps or atomic gaps of contaminated water, consistently and inconsistently. In some regions of the water, medium also conducts the current of partially survived photons in addition to deal with the dissipated heat energy. Due to this phenomenon, a body often faces the electric shock, which is due to photonic current instead of electric (or electronic) current.

A single cycle of electron dynamics of silicon atom generates the force and energy, which is termed as the unit photon. Thus, unit photon possesses the least length of conserved force and energy, where it has the shape like Gaussian distribution turned upwardly from both ends as shown in (1) of Figure 1; an inverted unit photon has shape like Gaussian distribution turned downwardly from both ends is also shown. In suitable configuration, interaction of the unit photon with electron results in its division into two equal parts (shaped like integral symbol and shaped like opposite integral symbol), which is related to one bit of energy in each case as shown in (2) and (3) of Figure 1, respectively. The heat energy is generated when a photon of length, even in the length of the unit photon, is divided by the interaction of electron.

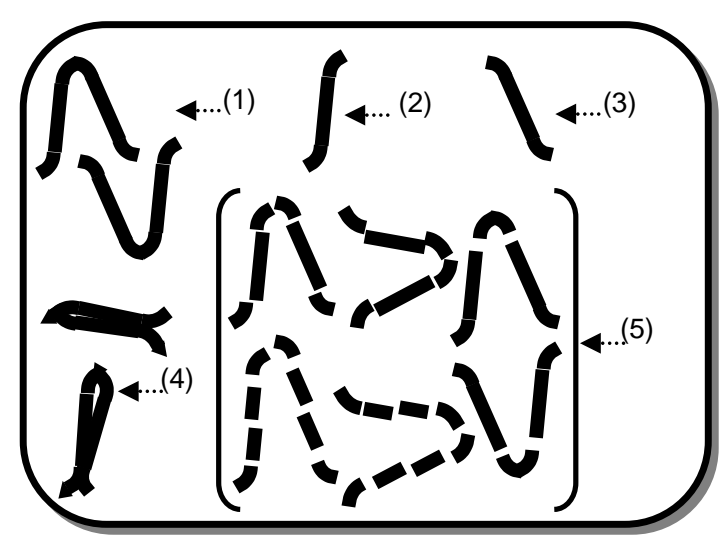

Figure 1: (1) The smallest entity of force and energy shape like Gaussian distribution turned from both ends is called the unit photon, the division of the unit photon in shape like (2) integral symbol and (3) opposite integral symbol, (4) merged energy of unit photon and (5) the division of unit photon into the tits and bits of heat 
When the unit photon interacts with suitable angle to clamped-energy-knot electron of the hypothesized solid atom despite its division into two, a merged energy is resulted as shown in (4) of Figure 1. A merged energy of unit photon is a bunch of folded energy. When the unit photon interacts with the side of clamped-energy-knot electron of the atom, it divides into several parts. Due to the dedicated interaction with the electron, unit photon divides into two parts, which can further sub-divide. Nevertheless, in the divided unit photon, where it is converted into tits and bits, it yet keeps a minute 'element of force' due to the turning positions of the electron under dynamics. In case where the arms of unit photon neither folded nor divided into two parts, the unit photon is divided into several parts as shown in (5) of Figure 1. They purely relate to tits and bits of heat.

The length of photon depends on the number of cycles carried out by the electron. If the interstate changing aspect of electron remained uninterrupted, the generation of photon also remained in the continuous length. A force and energy relationship that has shape like a wave is shown in (1) of Figure 2. This is called overt photon. It is generated by the three cycles of electron in forward and reverse directions.

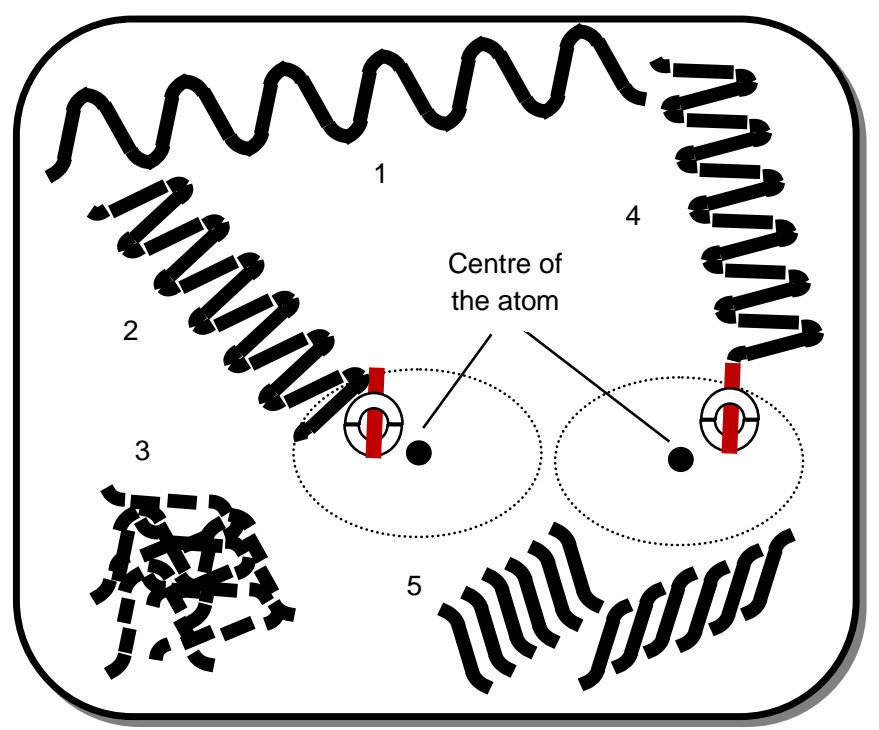

Figure 2: (1) An overt photon generated by the three cycles of electron dynamics in forward direction and reverse direction, (2) interaction of overt photon with the side of laterally orientated clamped-energyknot electron of the hypothesized solid atom, (3) tits and bits of heat resulted when that photon interacted with the side of laterally orientated clamped-energy-knot electron of the hypothesized solid atom, (4) interaction of overt photon with the tip of laterally orientated clamped-energy-knot electron of the 
hypothesized solid atom and (5) bits of energy shape like integral symbols resulted when photon interacted with the tip of laterally orientated clamped-energy-knot electron of the hypothesized solid atom

When a photon interacts with the side of clamped-energy-knot electron of the hypothesized atom under the suitable incidence, it is folded by the impact of absorption. This is shown in (2) of Figure 2. So, that overt photon converted into the tits and bits of heat as shown in (3) of Figure 2. Here, the element of force in pieces of tits and bits is almost diminished. The different pieces of that overt photon are now related to the heat energy.

By making the approximate angle of $90^{\circ}$ when the photon interacts with the tip of laterally orientated electron clamped by energy knot of the hypothesized solid atom, it divides into the bits of energy. This is shown in (4) of Figure 2. That overt photon converted into the integral symbols by dividing at the points of nodes and antinodes. This is shown in (5) of Figure 2, where single photon was converted into several pieces having the shape like integral symbols. A 'bit energy' is partially related to 'heat energy' as the (inside) force is still there to shape it.

As discussed for Figure 1, one bit of energy can further divide into the tits and bits of heat. It can be deduced that an overt photon is a combination of several unit photons, where division under miscellaneous interactions converted it into tits and bits of the heat energy. By combining two unit photons, an overt photon of least measured length is framed. Thus, an overt photon involves a minimum of two nodes and two antinodes.

\subsection{Photon energy - atomic and electronic scale phenomena}

In semisolid atoms, electrons deal with the zone of impartial force at $0^{\circ}$ orientation along the north-south poles [25]. So, when the electrons (of the outer ring) of semisolid atoms form orientation of $0^{\circ}$ along the normal lines drawn from the centers, they deal with an impartial force along the north-south poles. In silicon atom, electrons of the outer ring also keep half-lengths above the middle of clamped energy knot and half-lengths below the middle of clamped energy knots. So, electrons deal with force along the opposite tips at equal levels. It means that electrons also deal with the equal force along east and west poles. In this way, the electrons of the outer ring deal with the neutral behavior of 
the states. So, supply of the heat energy triggers their interstate dynamics where forces of all four poles remain conserved. This is not the case with electrons of the inner rings.

In Figure 3 (a), a top left-sided electron of silicon atom is studied to execute interstate dynamics. Here, electron involves the relevant forces exerting along the relevant poles. Forces of relevant poles of the electron introduce a 'moment of inertia' in auxiliary manner at each point of its turning. Energy of one bit is engaged along the traced trajectory in first half-cycle. Engaging energy wrapping the force shaping along the trajectory of that electron. Electron deals with the forces of relevant poles in a conserved manner where one bit energy engages to warp force shaping along the tracing trajectory. (A force is shaped from those sides of the electron, which do not face the exertion of dedicated forces.) That electron is lifted from the occupied state without touching to clamped energy knot. In silicon atom, electrons of the zeroth ring and the first ring do not entertain the exertion of forces. Only the electrons of the outer ring are capable to execute interstate dynamics in forward and reverse direction cycles.
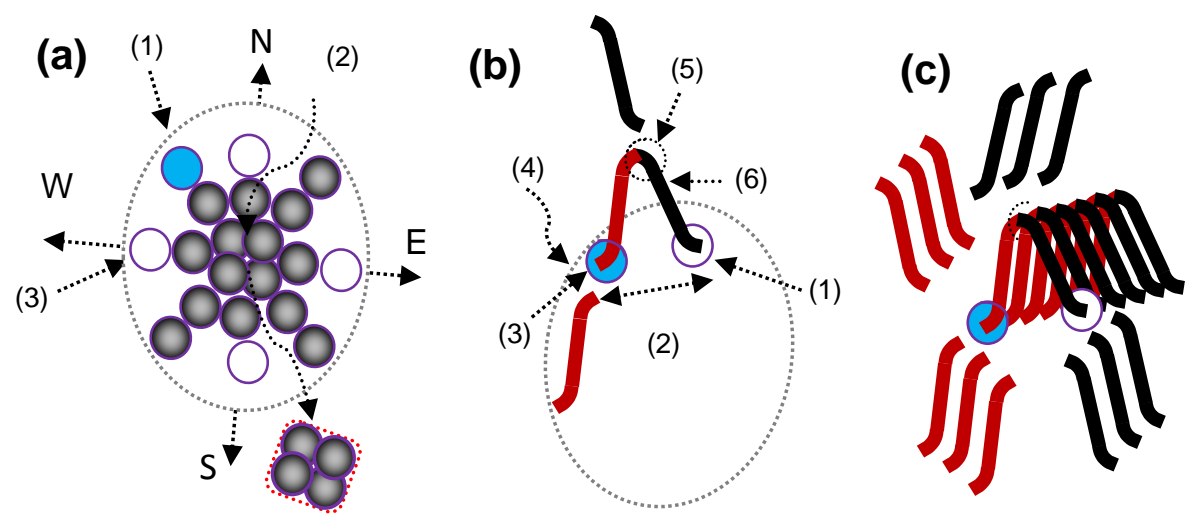

Figure 3: (a) Neutral-state silicon atom: (1) targeted electron; (2) zeroth ring; (3) unfilled energy knot.

(b) Electron dynamics of silicon atom in the forward direction cycle: (1) unfilled state; (2) interstate electron gap; (3) filled state; (4) bit energy is engaged to wrap force along the trajectory of electron in $1^{\text {st }}$ half cycle; (5) maximum limit point; (6) bit energy is engaged to wrap force along the trajectory of electron in $2^{\text {nd }}$ half cycle. (c) Overt photon of six unit photons having twelve bits of energy generated under the execution of interstate electron dynamics of three forward and three reverse direction cycles

In Figure 3 (b), the conversion of heat energy into the unit photon energy is shown. At the 'maximum limit point' of the lifted electron, one bit energy is engaged along the traced trajectory. Energy of the one bit wraps the force shaping along the trajectory of 
electron in the first half cycle. The trajectory of electron in first half cycle is up to the central point of the 'maximum limit point'. Here, the turning of the electron deals with the auxiliary 'moment of inertia'. In the second half cycle, another energy of one bit is engaged along the trajectory of electron to wrap the shaping force. The trajectory of electron in second half cycle is up to the last point of cycle. Here, again the turning of the electron deals with the auxiliary 'moment of inertia'. Thus, a unit photon is due to the force and energy that is generated from one complete forward direction cycle of interstate electron dynamics. That electron recalls 'moment of inertia' at each point of turning, which is in the auxiliary manner. A complete cycle of confined interstate dynamics of electron in forward direction is shown in Figure 3 (b), where the 'maximum limit point' of the electron is also shown.

Interstate dynamics of electron of the neutral-state silicon solid when completes the six cycles, three in the forward direction and three in the reverse direction, it shapes the energy of twelve bits in shape like a wave. Here, energy of six bits have the shape like the opposite integral symbol and energy of six bits have the shape like straight integral symbol as shown in Figure 3 (c). In the reverse direction cycle, electron deals with the exertion of forces in similar manner as in the case of forward direction cycle but according to the facing poles. In either cycle, electron does not make contact to (or with) the state of energy knot. So, under uninterrupted forward and reverse direction cycles, the execution of confined interstate dynamics of electron generates the forcing energy in connecting unit photons. The connecting shapes of the unit photons are just like that of continuous sketching on a strip of paper along with the pulling of paper simultaneously without picking pencil from the initial position.

In the semisolid atoms, electrons deal with half-length above and half-length below to the clamped energy knots [25]; in silicon atom, electrons remain orientated at zero degree along the north and south poles. Hence, a force is mechanically exerted along the relevant poles of electrons belonging to the outer ring of silicon atom. Force treats electrons of the outer ring only to execute dynamics. Changing aspects of an electron in the outer ring of silicon atom remain between the filled and unfilled states. In this way, a forced exertion along the poles of that electron remained in the conservative mode. 
When all four electrons of the outer ring executed confined interstate dynamics under the availability of heat energy of the silicon atom, they resulted in the generation of photon energy of four photons.

Heat energy of tits and bits engaging along the tracing trajectory in first half cycle of electron dynamics is equal to one bit energy. Heat energy equal to one bit energy is also engaged in the second half cycle of electron dynamics. So, force and energy of the unit photon generate in a cycle of forward direction or in a cycle of reverse direction. The overall shape of the engaged energy along the traced trajectory for the first half cycle dynamics of electron is like straight integral symbol $(\delta)$. The overall shape of the engaged energy along the traced trajectory for the second half cycle dynamics of electron is like opposite integral symbol (l). For left and right half cycles of electron dynamics, two shapes of integral symbols connect at the centre of 'maximum limit point' resulting in the overall shape of force and energy shape like Gaussian distribution turned from both ends as shown in Figure 4. The force and energy relationship along the trajectory of electron dynamics in the forward direction cycle is shown in Figure 4 (a); (1) to (6). The force and energy relationship along the trajectory of electron dynamics in the reverse direction cycle is shown in Figure 4 (b); (6) to (1). In Figure 4, the maximum limit point denoted by (7) in both trajectories falls exactly over the midpoint of interstate electron gap.

Figure 4: Sections of the unit photon generated by interstate electron dynamics of silicon atom in (a) forward direction cycle and (b) reverse direction cycle; (7) maximum limit point where left and right half cycles of electron dynamics connect
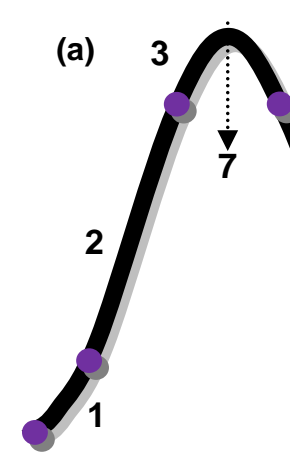

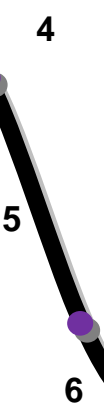

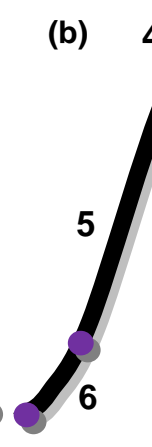

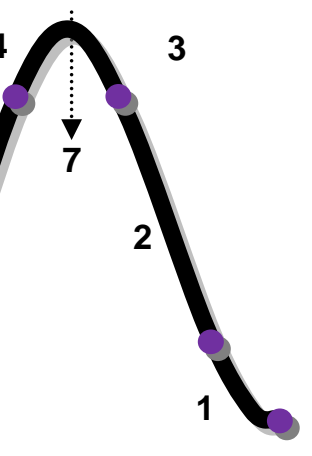

In the interstate electron dynamics of silicon atom, the energy of two bits is engaged in the forward direction cycle and the energy of two bits is also engaged in the reverse direction cycle. In this context, the resultant photon is termed as the overt photon of the 
least measured length. A continuous supply of heat energy to silicon atom will increase the length of forcing energy, i.e., the length of overt photon.

Positions of electron dealing with the auxiliary moment of inertia are responsible in forcing the energy of photon from one point to other. In this context, the exerting forces to different poles of electrons remained path-independent. However, exerting forces keep electrons of the outer ring confined within their interstate gap. Electrons do not possess any other way to re-gain. The motion to rest and rest to motion state of the electron at the centre of 'maximum limit point' is due to the diminishing of forcing exertion at that point, but electron is turning toward unfilled state due to the appearance of forcing exertion at that point. By recalling the 'moment of inertia' in the auxiliary manner, that electron deals with the forces. Forces of two poles act together but from the opposite sides, which causes that electron to turn. Thus, there is a coupling in terms of generation of force and energy of first half cycle dynamics and second half cycle dynamics. The recalled 'moment of inertia' is yet legible during less turning behavior of the electron. Hence, a path of electron under interstate dynamics in the less turning behavior is like '" or 'l'.

In Figure 5, electron of each quadrant (of the atom) shows the trajectory of executed interstate dynamics (both forward and reverse). In Figure 5 (a), an electron leaves the state from the rear side (tail) and enters the nearby state from the front side (head) in forward direction interstate dynamics, so it leaves the state from the rear side (tail) and enters to the nearby state from the front side (head) in reverse direction interstate dynamics. An opposite is the case for electron shown in Figure 5 (b). In Figure 5 (c), an electron leaves the state from the front side (head) and enters to the nearby state from the rear side (tail) in forward direction interstate dynamic, so it leaves the state from the front side (head) and enters to the nearby state from the rear side (tail) in reverse direction interstate dynamic. An opposite is the case for electron shown in Figure 5 (d). So, electrons of confined interstate dynamics just execute the rise and fall in Figure (a) and (d), and the fall and rise in Figure 5 (b) and (c) by considering the associated energy knots as the reference points. In forward and reverse direction cycles, forces involved in the dynamics of electrons at different positions or points are shown 
symbolically in Figure 5 (a-d); red arrows are related to the forward direction cycle of electron dynamics and black arrows are related to the reverse direction cycle of electron dynamics.

(a) (E, N)

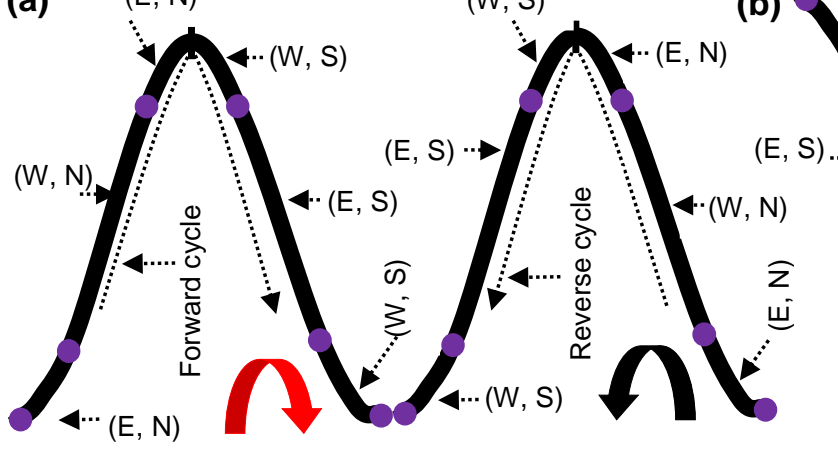

(b) $\cdots(W, S)$

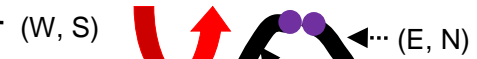

$(W, S)$

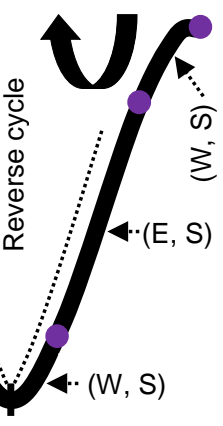

$(\mathrm{E}, \mathrm{N})$

$(\mathrm{W}, \mathrm{S})$
$(E, N)$

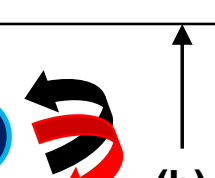

(b)

\section{Neutral-state silicon atom}

(a)

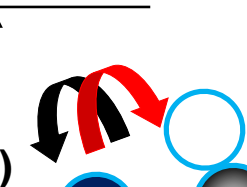

$\mathbf{N}$
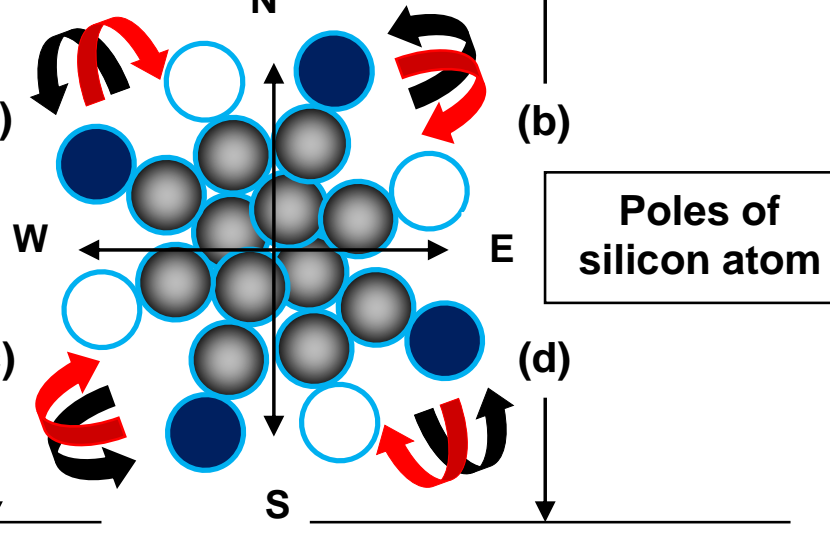

(d)

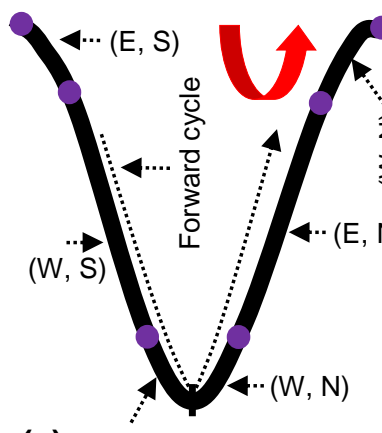

(c)

(E, S)

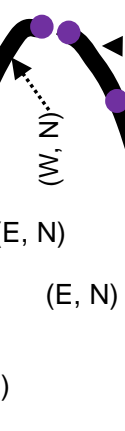

$(\mathrm{W}, \mathrm{N})$

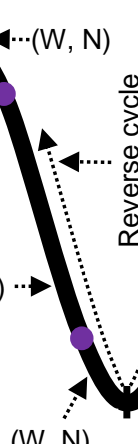

$(N, N)$

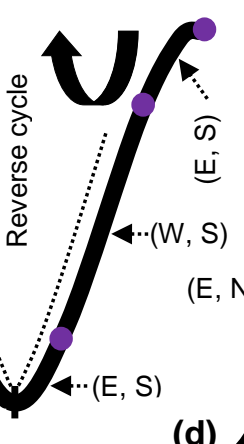

(d)
$(\mathrm{W}, \mathrm{N})$

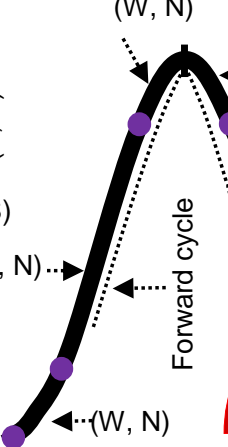

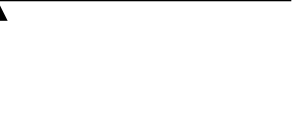

Poles of con atom

Figure 5: Electrons (blue color) deal with the forces along relevant poles while executing confined interstate dynamics of forward direction cycle (denoted by red colored turning symbol) and reverse direction cycle (denoted by black colored turning symbol) are shown in the respective quadrants (a), (b), (c) and (d); forces of east $(E)$, west $(W)$, north $(N)$ and south $(S)$ poles exerted along the relevant poles of electrons of silicon atom are labelled in the trajectories of unit photons 
A propagating photon through the interstate electron gaps deals with the forcing energy in a wave like fashion. There are other shapes of photons, where the forcing energy behaves differently. In atoms of suitable elements, where four conservative forces are involved for the electron to undertake inter-state dynamics, they transform heat energy into the photon energy having the shape like a wave. In the atoms of those elements, where three conservative forces are involved, interstate electron dynamics transform heat energy into the photon energy having the shape like a connected integral symbols. In the atoms of those elements, where two conservative forces are involved, interstate electron dynamics transform heat energy into the photon energy having the shape like a connected tick symbols. Heat energy converts into the photon energy having the shape like a connected ' $L$ ' alphabets in atoms where two conservative forces involves in such a manner that an electron attempts to cross the pole of its atom while in the dynamics. Photons shapes like connected integral symbols, tick symbols and $L$-like symbols are shown in Figure 6 (a), (b) and (c), respectively. In Figure 6 (d), a generating photon shows both the elements of force and energy.

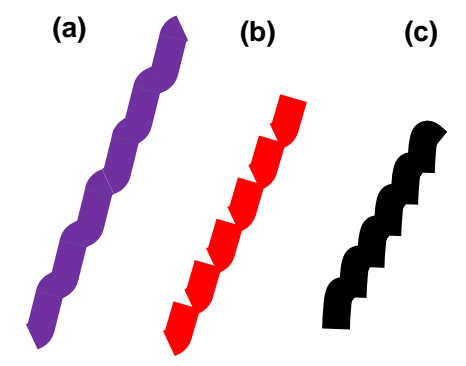

(d)

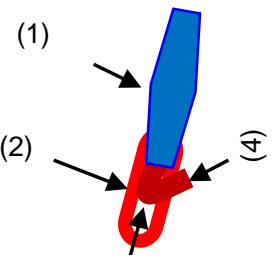

(3)

Figure 6: An overt photon of connected (a) integral symbols, (b) tick symbols, (c) L-like symbols and (d) electron shaped force and energy along the trajectory of the confined dynamics; (1) electron of interstate dynamics, (2) wrapped energy, (3) force, (4) cropped portion of wrapped energy showing the element of force inside

\subsection{General discussion}

In the solar panel, each silicon cell connected in the series adds up to the generating number of photons under the suitable fabrication procedure. The heat dissipation at the rear side surface of solar cell is controlled by using the silver paste or through other 
means. The generating photons are collected at the suitable sides or points in the laminated panels. As observed in solar panel, it can generate the maximum power when setting is under the suitable inclination. The cycles of confined interstate electron dynamics of silicon atoms remain uninterrupted for a longer period, where on titling silicon solar panel at suitable orientation with respect to base results in varying the efficiency. Depositing silicon atoms in few layers at a suitable substrate can generate the high power instead of depositing several layers in microns size.

When an overt photon interacts with suitable electron of the hypothesized solid atom by making an approximate angle of $90^{\circ}$, it is mainly converted into the bits of energy where each unit photon is divided into the energy of two bits. The bits of energy surround atoms of silicon lattice on entering the laminated glass. So, bits of energy process to work for the heat energy. However, when that overt photon interacts with the side of clamped electron, that photon converts into tits and bits of heat. When featured photons interacted with the tips of laterally orientated electrons of elongated atom by making the suitable angle, reverted element of the force prints the pattern [28]; photons print the effects in the form of dots for multidimensional particles and print the effects in the form of lines for one-dimensional particles.

Where there is no specific interaction of a photon with matter (at atomic level or electronic level), it divides into the tits and bits of heat. Heat energy of a divided photon dissipate in the structure based on atoms and electrons. However, the resulted heat energy of divided photon can inspire by wrapping the force shaping along the trajectory of an electron once a suitable atom of the structure enables the execution of interstate electron dynamics. In this context, the conversion of one form of energy into other form of energy depends on the type and characteristics of the structure.

An amorphous material do not have the ordered structure. When the photons of featured X-rays interact with such type of material by making the suitable angles, energy of photons mainly converts into the heat energy. Here, the XRD scan is not obtained with discernable peaks of the structure. In some cases, photons having characteristics of current are utilized to split the matter like inert gas atoms [24]. 
The set modalities of photons depend on the origin of generation, so establish the roles set by the manufacturer. In this context, structural design is crucial to target the specific application and many studies are now targeted to explore the structure [29-38]. However, it has been disclosed elsewhere [18] that size and shape of the structure in various metallic colloids are owing to the controlled behavior of force and energy [18]. However, this is different in the structures of semisolid atoms [21, 39].

In atoms of suitable elements, when executed electron dynamics is for one forward cycle or for one reverse cycle, a generated energy in particular shape binds the atoms [26]. But, when executed electron dynamics is for many forward cycles and many reverse cycle, photons of different shapes are generated as shown in Figure 6 (a-c). When an atom of tiny-sized particle has the deteriorated interstate electron dynamics, it can work defectively rather than effectively for the chosen application of nanomedicine [40]. The development of particles under predictor packing is also studied, where photons shaped like wave converted into the tuned pulses to optimize the process [41]. To measure the temperature of such materials is an integral part and some of the studies also shed light on it [42-44]. A recent study explained the role of van der Waals interactions in isolated atom by considering the induced dipoles [45] which can be attained when fluctuations of charge density are in wave-like nature [46].

\section{Conclusion}

Heat energy wraps the force shaping along the trajectory of electron in dynamics. The force shapes from the sides of electron not facing the exertion of dedicated forces. A unit photon is generated for one forward or reverse interstate electron dynamics, which has the shape like Gaussian distribution turned from both ends. A continued length photon is generated by the electron dynamics of silicon atom, if heat energy is available.

When suitable population of the photons propagates in the interstate electron gaps, they work as a photonic current [24]. Photon energy gets converted into the heat energy when interacted with the electron of hypothesized solid atom. When a photon interacts with the side of laterally orientated electron clamped by energy knot of the atom, it gets divided into the tits and bits of heat. When a photon interacts with the north sided tip of 
laterally orientated electron by making the approximate angle of $90^{\circ}$, it gets divided into the bits of energy. Bits of energy further get divided into the tits and bits of heat.

Upon executing the interstate electron dynamics, a suitable atom transforms the heat energy into the photon energy. A unit photon involves energy of two bits only, whereas a continued length photon involves energy of the several bits. A photon when in the least measured length, it forms by the connection of at least two unit photons.

The exerting forces to electron change the aspects by restricting it in the interstate gap. Supplied heat energy to the atom wraps the force shaping along the trajectory of electron executing interstate dynamics. In this way, the force shaping along the trajectory of an electron preserves the features. So, generated force and energy remain conserved; first force involves to exert along the poles of electron, instantaneously energy engages to wrap the force shaping along the trajectory of electron undertaking dynamics. This is how a photon is formed. So, in suitable neutral-state atoms, the charisma of conserved forces enables the charisma of conserved energy.

Electron of the outer ring of silicon atom deals with forces along the relevant poles in such a manner that it recalls auxiliary moment of inertia at each point of the turning. (An outer ring means the outer most ring of the atom.) The centre of the silicon atom acts as the reference point of electrons of the outer ring executing confined interstate dynamics.

The exerting force to electron functions only in the width of photonic band gap or interstate electron gap of the silicon atom. Before crossing the 'maximum limit point', the electron is examined by the opposite forces to pull it downward. First the electron of outer ring gets lifted in a lateral manner by turning upward. To turn it back, it gets relief from the effect of upward force to join exerting force in the adjacent manner. So, in order to turn downward, a force in (opposite) lateral manner is exerted to electron. In fact, forces exerting to electron in acquiring positions of different lateral and adjacent orientations are as per their available viability. Under the conservative force, speed of electron in confined interstate dynamics approaches almost near to the speed of light.

Depending on the built-in interstate gap of electron dynamics, different atoms generate different shaped photons. In addition to shape like waves, photons shape like connected ticks, integral symbols and ' $L$ ' symbols are also shaped. 
In neutral-state semisolid atom, electrons deal with zero degree orientation along the normal lines drawn from the centers, which is not the case in atoms of gaseous and solid states as discussed in reference 25 . A mechanism of photon generation indicates that atomic structure is different. The mechanism of heat energy conversion into photon energy shows that atoms have different shapes.

Now, a photon has a strong analogy to electron in such a manner that the latter forms a photonic band gap (or interstate gap) enabling the former to propagate through the gap to work as a photonic current. A travelling photon transports the forcing energy from one end to the other end. Photons interact with the matter to dissipate the heat energy and to permeate the force. When a photon interacts with matter by making a suitable angle, it either divides into tits and bits or divides into bits of energy.

Electrons impinge on atoms, so atoms deal with the modification [24]. Electrons possess the mass to deal with energy and force when atoms in transition [25]. Heat energy and photon energy are not the ones that impinge or deal with the energy and force. Energy can keep the force to behave impartially in exertion for it [27].

\section{References}

[1] D. Bohm, D. Pines, A Collective Description of Electron Interactions. I. Magnetic Interactions, Phys. Rev. 82, (1951) 625-634.

[2] D. Pines, D. Bohm, A Collective Description of Electron Interactions: II. Collective vs Individual Particle Aspects of the Interactions, Phys. Rev. 85, (1952) 338-353.

[3] R. H. Ritchie, Plasma Losses by Fast Electrons in Thin Films, Phys. Rev. 106, (1957) 874-881.

[4] D. Bohm, D. Pines, A Collective Description of Electron Interactions: III. Coulomb Interactions in a Degenerate Electron Gas, Phys. Rev. 92, (1957) 609-625.

[5] T. Low, et al., Polaritons in layered two-dimensional materials, Nat. Mater. 16, (2017) 182-194.

[6] J. Frenkel, On the Transformation of Light into Heat in Solid. I, Phys. Rev. 37, (1931) 17-44. 
[7] O. D. D. Couto Jr. et al., Charge control in $\operatorname{InP} /(G a, I n) P$ single quantum dots embedded in Schottky diodes, Phys. Rev. B 84, (2011) 125301-7.

[8] M. Brust, M. Walker, D. Bethell, D. J. Schiffrin, R. Whyman, Synthesis of Thiolderivatised Gold Nanoparticles in a Two-phase Liquid-Liquid System, J. Chem. Soc., Chem. Commun. (1994), 801-802.

[9] R. L. Whetten, J. T. Khoury, M. M. Alvarez, S. Murthy, I. Vezmar, Z. L. Wang, P. W. Stephens, C. L. Cleveland, W. D. Luedtke, U. Landman, Nanocrystal Gold Molecules, Adv. Mater. 8, (1996) 428-433.

[10] M. Brust, C. J. Kiely, Some recent advances in nanostructure preparation from gold and silver particles: a short topical review, Colloids and Surfaces A: Physicochem. Eng. Aspects 202, (2002) 175-186.

[11] S. C. Glotzer, M. J. Solomon, Anisotropy of building blocks and their assembly into complex structures, Nature Mater. 6, (2007) 557-562.

[12] S. Link, M. A. El-Sayed, Shape and size dependence of radiative, nonradiative and photothermal properties of gold nanocrystals, Int. Rev. Phys. Chem. 19, (2000) 409- 453.

[13] C. P. Shaw, D. G. Fernig, R. Lévy, Gold nanoparticles as advanced building blocks for nanoscale self-assembled systems, J. Mater. Chem. 21, (2011) 12181-12187.

[14] Y. Negishi, T. Nakazaki, S. Malola, S. Takano, Y. Niihori, W. Kurashige, S. Yamazoe, T. Tsukuda, H. Häkkinen, A Critical Size for Emergence of Nonbulk Electronic and Geometric Structures in Dodecanethiolate-Protected Au Clusters, J. Am. Chem. Soc. 137, (2015) 1206-1212.

[15] A. Moscatelli. Gold nanoparticles: Metallic up to a point, Nature Nanotechnol. (2015), DOI:10.1038/nnano.2015.16.

[16] M. Ali, I -Nan. Lin, Effects of the Electronic Structure, Phase Transitions and Localized Dynamics of Atoms in the Formation of Tiny Particles of Gold. (2020), http://arxiv.org/abs/1604.07144 (last version)

[17] M. Ali, I -N. Lin, Development of Gold Tiny Particles and Particles in Different Sizes at Varying Precursor Concentration. Adv. Nat. Sci: Nanosci. Nanotechnol. 11, (2020) 015006 (13pp). 
[18] M. Ali, I $-\mathrm{N}$. Lin, Controlling morphology-structure of gold tiny particles, nanoparticles and particles at different pulse rates and pulse polarity. Adv. Nat. Sci: Nanosci. Nanotechnol. 10, (2019) 025015 (14pp).

[19] M. Ali, I $-\mathrm{N}$. Lin, Formation of tiny particles and their extended shapes: origin of physics and chemistry of materials. Appl. Nanosci. 9, (2019) 1367-1382.

[20] M. Ali, I -N. Lin, C. -J. Yeh, Tapping Opportunity of Tiny-Shaped Particles and Role of Precursor in Developing Shaped Particles, NANO 13, (2018) 1850073 (16 pages).

[21] M. Ali, I - N. Lin, Phase transitions and critical phenomena of tiny grains carbon films synthesized in microwave-based vapor deposition system. Surf. Interface Anal. 51, (2019) 389-399.

[22] M. Ali, M. Ürgen, Switching dynamics of morphology-structure in chemically deposited carbon films -A new insight, Carbon 122, (2017) 653-663.

[23] M. Ali, Tiny-Shaped Particles Developing a Mono Layer Shape Dealing with Localized Gravity and Levity at the Solution Surface. (2020), http://arxiv.org/abs/1609.08047 (last version)

[24] M. Ali, Atoms of None of the Elements Ionize While Atoms of Inert Behavior Split by Photonic Current. (2020), http://arxiv.org/abs/1611.05392 (last version)

[25] M. Ali, Atoms in Gaseous and Solid States and their Energy and Force Relationships under Transitional Behaviors. https://doi.org/10.21203/rs.3.rs$\underline{88120 / v 1}$ (2020)

[26] M. Ali, Structure Evolutions in Atoms of the Elements Executing Confined Interstate Electron Dynamics. (2020), http://arxiv.org/abs/1611.01255 (last version)

[27] M. Ali, Atomic Structure and Binding of Carbon Atoms. https://www.preprints.org/manuscript/201801.0036/v10

[28] M. Ali, I -N. Lin, Gold Nanostructures and Microstructures with Tunable Aspect Ratios for High-Speed Uni- and Multidirectional Photonic Applications. (2020), ACS Appl. Nano Mater. 3 (9), (2020) 9410-9424.

[29] J. Zhao, L. Yang, Structure Evolutions and Metallic Transitions in $\ln _{2} \mathrm{Se}_{3}$ Under High Pressure, J. Phys. Chem. C 118, (2014) 5445-5452. 
[30] V. N. Manoharan, Colloidal matter: Packing, geometry, and entropy, Science 349, (2015) 1253751.

[31] J. Park, et al., 3D structure of individual nanocrystals in solution by electron microscopy, Science 349, (2015) 290-295.

[32] D. Jacobsson, et al., Interface dynamics and crystal phase switching in GaAs nanowires. Nature 531, (2016) 317-322.

[33] T. Tuma, A. Pantazi, M. L. Gallo, E. Eleftheriou, Stochastic phase-change neurons. Nature Nanotech. 11, (2016) 693-699.

[34] J. Rensberg, et al., Active Optical Metasurfaces Based on Defect-Engineered Phase-Transition Materials, Nano Lett. 16, (2016) 1050-1055.

[35] Y. Suzuki, G. Cardone, D. Restrepo, P. D. Zavatteri, T. S. Baker, F. A. Tezcan, Selfassembly of coherently dynamic, auxetic, two-dimensional protein crystals, Nature 2016, 533, 369-373.

[36] C. H. J. Evers, J. A. Luiken, P. G. Bolhuis, W. K. Kegel, Self-assembly of microcapsules via colloidal bond hybridization and anisotropy, Nature 534, (2016) 364368.

[37] I. R. Epstein, B. Xu, Reaction-diffusion processes at the nano- and microscales. Nature Nanotech. 11, (2016) 312-319.

[38] A. Azizi, Spontaneous Formation of Atomically Thin Stripes in Transition Metal Dichalcogenide Monolayers, Nano Lett. 16, (2016) 6982-6987.

[39] M. Ali, M. Ürgen, Morphology and Structure of Carbon Films Deposited at Varying Chamber Pressures. (2020), https://arxiv.org/abs/1802.00730 (last version)

[40] M. Ali, Nanoparticles-Photons: Effective or Defective Nanomedicine, J Nanomed Res. 5, (2017) 00139.

[41] M. Ali, I -N. Lin, C. -J. Yeh, Predictor Packing in Developing Unprecedented Shaped Colloidal Particles, NANO 13, (2018) 1850109 (15 pages).

[42] M. Mecklenburg, W. A. Hubbard, E. R. White, R. Dhall, S. B. Cronin, S. Aloni, B. C. Regan, Nanoscale temperature mapping in operating microelectronic devices, Science 347, (2015) 629-632. 
[43] L. Ye, D. Hou, X. Zheng, Y. Yan, M. D. Ventra, Local temperatures of stronglycorrelated quantum dots out of equilibrium, Phys. Rev. B 91, (2015) 205106-8.

[44] F. Menges, et al., Temperature mapping of operating nanoscale devices by scanning probe thermometry, Nat. Commun. 7, (2016) 10874 doi: 10.1038/ncomms 10874.

[45] S. Kawai, et al., Van der Waals interactions and the limits of isolated atom models at interfaces, Nat. Commun. (2016) DOI: 10.1038/ncomms11559.

[46] A. Ambrosetti, N. Ferri, R. A. DiStasio Jr., A. Tkatchenko, Wavelike charge density fluctuations and van der Waals interactions at the nanoscale, Science 351, (2016) 1171-1176.

\section{Author's biography:}

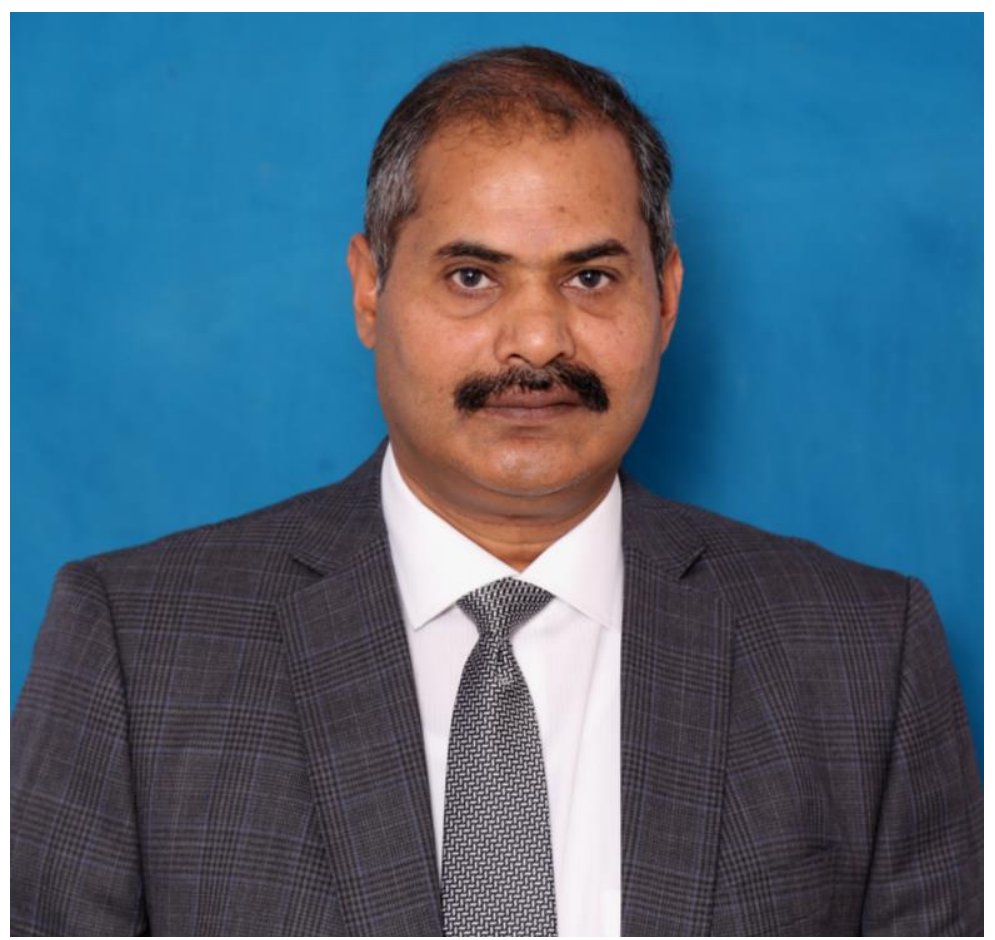

Mubarak Ali graduated from University of the Punjab with BSc (Phys \& Maths) in 1996 and MSc Materials Science with distinction from Bahauddin Zakariya University, Multan, Pakistan (1998); his thesis work completed at Quaid-i-Azam University Islamabad. He gained PhD in Mechanical Engineering from the Universiti Teknologi Malaysia under the award of Malaysian Technical Cooperation Programme (MTCP;2004-07) and postdoc in advanced surface technologies at Istanbul Technical University under the foreign fellowship of The Scientific and Technological Research Council of Turkey (TÜBITAK, 2010). 
He completed another postdoc in the field of nanotechnology at the Tamkang University Taipei (20132014) sponsored by National Science Council now M/o Science and Technology, Taiwan (R.O.C.). Presently, he is working as Assistant Professor on tenure track at COMSATS University Islamabad (previously known as COMSATS Institute of Information Technology), Islamabad, Pakistan (since May 2008) and prior to that worked as assistant director/deputy director at M/o Science \& Technology (Pakistan Council of Renewable Energy Technologies, Islamabad, 2000-2008). He was invited by Institute for Materials Research, Tohoku University, Japan to deliver scientific talk. He gave several scientific talks in various countries. His core area of research includes materials science, physics \& nanotechnology. He was also offered the merit scholarship for the PhD study by the Higher Education Commission, Government of Pakistan, but he did not avail himself of the opportunity. He also earned Diploma (in English language) and Certificate (in Japanese language) in 2000 and 2001 respectively, in part-time from the National University of Modern Languages, Islamabad. He is the author of several articles available at following links; https://scholar.google.com.pk/citations?hl=en\&user=UYjvhDwAAAAJ, https://www.researchgate.net/profile/Mubarak Ali5, https://www.mendeley.com/profiles/mubarakali7/, \& https://publons.com/researcher/2885742/mubarak-ali/publications/ 